\title{
Presence of emerging Salmonella spp. serovars in pig farms: a risk to public health
}

\begin{abstract}
The protection of consumers from emerging Salmonella spp. serovars depends on surveillance and control of the pathogen in food producing animals. Among them, pigs are the second most important species for transmitting emerging Salmonella spp. serovars pathogenic to consumers. Thus, a microbiological investigation of pig salmonellosis was undertaken in Central Greece examining samples collected from multiple carcass sites, aiming at the identification of prevalent serovars and their carcass location. For this purpose 492 tissue samples and 378 environmental swab samples were cultured following ISO 6579:2002. Twelve different serovars were isolated from feacal and tissue samples and two from the environmental samples, representing a total of 79 isolates. Among the identified serovars were the emerging monophasic Typhimurium serovars, $S$. enterica subsp. enterica ser. 4,5, 12:i:- (16 isolates) and $S$. enterica subsp. enterica ser. 4,12:i:- (7 isolates), the monophasic $S$. enterica subsp. enterica ser. 6,7:k:- (6 isolates) and the rarer serovars of subspecies enterica subsp. diarizonae 61:k:1,5, and $S$. enterica subsp. houtenae 40:g,t:(one isolate each). Of the remaining isolates, 37 were serovar Typhimurium, six (6) were serovars S. Bredeney (2 isolates), S. Agona, S. Derby, S. Infantis, S. Meleagridis, and S. Cerro and four were untypeable. Although serovar Typhimurium was the most prevalent one (37 isolates), the other serovars could also be considered of emerging public health importance, due to pork contamination. Thus, the pig appears in Greece as a possible source of human salmonellosis caused not only by commonly reported serovars, but also of rarer ones.
\end{abstract}

Keywords: pig, salmonellosis, slaughterhouse, tissue
Volume 7 Issue 3 - 2018

\author{
Grammato Evangelopoulou,' Georgios \\ Filioussis, ${ }^{2}$ Spyridon Kritas, ${ }^{2}$ Anastasios \\ loannidis, ' Angeliki R Burriel' \\ 'Department of Nursing, University of Peloponnese, Greece \\ ${ }^{2}$ Department of Microbiology and Infectious Diseases, Aristotle \\ University, Greece
}

\begin{abstract}
Correspondence: Grammato Evangelopoulou, Laboratory of Integrated Healthcare, Department of Nursing, Faculty of Human Movement and Quality of Life Sciences, University of Peloponnese, Sparta 23 I00, Greece, Tel 30-694-584-7840, Email matinavet@hotmail.com
\end{abstract}

Received: February 26, 2018 | Published: June 06, 2018

\section{Introduction}

Salmonella spp. are the commonest cause of foodborne enteric disease in man and the second most frequently reported zoonotic agent in the $\mathrm{EU}^{1} \mathrm{~A}$ mean $10-20 \%$ of human infections ${ }^{2}$ across EU are attributed to the consumption of pork products, although in some member states this may reach $56 \%{ }^{3}$ Thus, the contribution of pork products to human infections appears high enough to make control of pig samonellosis an objective of the pig industry. Effective control of pig salmonellosis depends on measures preventing the pathogen of entering a pig farm. Pigs usually get infected through the faecal-oral route; hence carrier animals (pigs or other species) should be prevented of entering the premises. After infection of the host, the pathogen initially colonizes the intestine and eventually the gut-associated lymphoid tissue and other organs of the digestive tract. From these tissues it is intermittently excreted into the gut and eventually the animal's environment. ${ }^{4,5}$ In these events, important role play the lymph follicles (Peyer's patches) of the ileum wall. ${ }^{6-8}$ Peyer's patches are the main source of Salmonella serovars infecting other organs of the host, such as the lymph nodes and the gallbladder. ${ }^{9}$ Colonization of the mesenteric lymph nodes and gallbladder epithelium may result to the survival of various highly infective serovars, although the infected pigs usually remain asymptomatic. ${ }^{10,11}$ Pigs subclinically infected with Salmonella spp. are confirmed as carriers only after multisite carcass sampling and microbiological examination. This procedure is time consuming and cost ineffective, thus not routinely used. Hence, subclinically infected pigs, remaining largely unidentified, are a potential source of infection for other animals and humans. Furthermore, they are contributors to the development of an endemic 'house flora' of Salmonella within the slaughterhouse contaminating carcasses $^{12-15}$ and eventually pork consumers. A strong correlation has been found between the proportion of animals with Salmonella positive feaces and contamination of carcasses. An estimated 70\% of carriers are responsible of their own carcass contamination, while contaminated equipment, such as the carcass splitter, or the manual handling of carcasses are responsible for 15 to $30 \%$ of contamination. ${ }^{12,16}$ Contaminated slaughterhouse equipment is more important than handlers for carcass contamination, due to bacterial growth during the day inside or on the surface of equipment. ${ }^{17}$ Thus, by minimizing positive animals entering the slaughterhouse one minimizes the building up of an endemic "house flora" positively contributing to the protection of consumers. Therefore, the objectives of the present study were the identification of Salmonella serovars present in Greek swine farms, their carcass location and the discussion of the findings in relation to information reported from human cases occurring worldwide.

\section{Material and methods}

\section{Herd information and sample collection}

Data considered significant for the presence of Salmonella in pig farms of central Greece ${ }^{18}$ were collected through a number of questions answered by 15 farm owners agreeing to participate in the study. Sampling of finishers from this furrow-to- finish herds, having 20 to 500 sows each, was performed with regular visits to two slaughterhouses of the same area. Four hundred ninety two (492) tissue samples were collected from 123 randomly selected pigs. An estimated $5 \%$ of the fatteners (about 2500) slaughtered during the sampling period (September 2013 - March 2014) were microbiologically examined. Tissue samples collected after evisceration were from the colon, ileum, mesenteric lymph nodes and muscle from the pigs' neck (site of carcass drainage). Environmental samples were collected at 
the day of sampling carcasses. In each sampling day three sampling rounds were performed: one before slaughtering and two during carcass processing. The samples collected were from the hands and knives of two skinners of the dirty zone and three eviscerators of the clean zone and the carcass splitter. The knife blade was swabbed from tip to base twice on both sides, as was also the blade from the splitter. Each swab sample of the gloves was collected by swabbing the whole palm surface of both hands. In total, 378 environmental samples were collected.

\section{Isolation and serotyping of Salmonella spp.}

The Salmonella ISO 6579:2002, ${ }^{19}$ Annex D for food and animal feeding stuffs ${ }^{19}$ was strictly followed for the isolation of Salmonella serovars. The selected "choice laboratory medium" were SalmonellaShigella agar (SS Merck- Germany) and Brilliant Green agar (BG) (CM329; Oxoid, England). Thus, three selective media were included in the isolation procedure. Suspect colonies on these media were subcultured on Columbia blood agar (Oxoid, England). They were examined with Gram stain and if they were Gram negative rods, they were tested for oxidase production and the utilization of Triple Sugar Iron Agar (Merck- Germany). Two biochemical systems, API 20E (Biomerieux, France) and the MicrogenTM GnA+B-ID (Microgen Bioproducts Ltd, UK) suitable for Gram (-) bacteria were used for assigning isolates to species. The isolates identified as Salmonella $s p p$. were further tested, with a polyvalent slide agglutination test (Remel Europe Ltd; Dartford, England) detecting O- and H- antigens. If they were positive, they were sent to the Greek National Reference Laboratory (GNRL) for specific serotyping.

\section{Results}

\section{Prevalence of Salmonella and herd conditions}

Only $3(20 \%)$ of the 15 herds examined were culture negative in all types of tissue samples collected. They were small herds having about 40 sows each and not reporting a recent entrance of replacement animals. Their owners did not report prophylactic use of antimicrobial agents, but they reported whitewashing between batches in addition to common cleaning and disinfection procedures. Herds above 100 sows, purchasing replacement animals and using antimicrobials for prophylactic purposes had slaughtered animals infected in at least one carcass site. One of these farms used also acidifiers in weaners' feed, as a preventing measure for Salmonella infections. In total, 39 (31.7\%) pigs of the 123 sampled were found Salmonella positive, but only nine $(23 \%)$ had more than one tissue sample positive. Specifically, 18 (46.15\%) had a positive ileum, 17 (43.6\%) had infected mesenteric lymph nodes, $11(28.2 \%)$ had positive feacal samples, and $6(15.38 \%)$ had positive neck muscle (Table 1). The last were all from pigs having at least one more positive tissue. Twenty seven (7.4\%) of the 378 environmental samples were found positive. Positive samples derived from multiple samplings of the non-disposable gloves of one of the skinners.

\section{Serovars identified}

A total of 79 isolates ( 52 from tissue and 27 from the environment) were identified as Salmonella spp. by the API, Microgen systems and the polyvalent slide agglutination test and they were sent to the GNRL for further serotyping. Of the 39 positive pigs (Table 1), one was infected with two different serovars from two different sampling sites. Twelve different Salmonella spp. serovars were identified. They were S. Typhimurium (20 isolates), the monophasic S.enterica subsp. enterica ser. 4,12:i:- (7 isolates), S.enterica subsp. enterica. 6,7:k:- (6 isolates), S.enterica subsp. enterica ser. 4,5, 12:i:- (6 isolates) serovars S. Bredeney (2 isolates) and one each S. Agona, S. Derby, S. Infantis, S. Meleagridis, S. Cerro, S. enterica subsp. diarizonae 61:k:1,5, and S. enterica subsp. houtenae 40:g,t:-. Four isolates were not serotyped and registered as 'rough strains'. The twenty seven serovars of the positive environmental samples were $S$. Typhimurium (17 isolates) and the monophasic S.enterica subsp. enterica ser. 4,5,12:i:- (10 isolates). They were isolated repeatedly from the same non-disposable gloves of one skinner, at all sampling dates.

\section{Discussion}

The present investigation revealed a large range of identified serovars (12 different serovars) among pigs of Greek farms. The range of identified serovars is, perhaps, evidence of Salmonella serovars spreading across EU, due to free trade of livestock and feed stuff. Greek pig farms are mostly receiving their replacement stock from EU breeders. ${ }^{20}$ The identified serovars belong to three of the six recognized Salmonella subspecies. This variability has not been reported previously from Greek pig farms, ${ }^{21,22}$ perhaps, due to restricted carcass site sampling or changes due to live pigs arriving in Greek herds from a variety of community sources and spreading to pork consumers across EU. This is, perhaps, the reason that although the most prevalent serovar was Typhimurium (Table 1), recognized in EU as one of the serovars frequently associated with human and pig salmonellosis, ${ }^{23}$ an equivalent number was emerging serovars not reported previously from Greek swine farms. ${ }^{21}$

Among the emerging serovars were monophasic variants of $S$. Typhimurium, $S$. enterica ser. 4,5,12:i:- and $S$. enterica ser. 4,12:i:, found here to be the second most prevalent serovars (Table 1). These variants have recently increased in prevalence among consumers, replacing gradually other important serovars associated to swine and pork products. ${ }^{24-27}$ Interestingly, these variants were not isolated from feaces in the present study due, perhaps, to their adaptation to the tissue of other digestive tract organs, such as the gallbladder ${ }^{9}$ and lymph nodes. At such organs, they remain dormant until stressful factors cause their multiplication and subsequent gut and environmental contamination, putting at risk other animals and eventually consumers. Such adaptation could also be responsible for the increased reporting of these variants from animals only recently, coinciding with carcass multisite sampling rather than just feaces and environmental sampling. The increased frequency of their isolation from human cases in different countries is, perhaps, a result of this adaptation. ${ }^{28-30}$ Hence, consumers are, perhaps, infected through consumption of undercooked meat harboring the pathogen in lymphatic tissue, rather than meat contaminated during its processing e.g. at slaughtering. This was evident from the positive neck muscle tissue deriving only from animals having at least one more positive site.

Monophasic S. Typhimurium or "S. Typhimurium-like" variants are currently in the fourth place among important serovars reported from man, in the second reported from live pigs and in the third reported from pig meat. They have also been isolated from a number of other animal species, such as poultry, cattle, turtles and food products, such as dried pork sausages. ${ }^{29,25,31,32}$ They show very high molecular relatedness to the 'classic' S. Typhimurium, a reason for possible misclassifications without studies of molecular relatedness. 
Thus, molecular relatedness, in respect to pathogenicity genes, helps the avoidance of misclassifications, but it also changes the epidemiological importance of newly emerging serovars from animals and consumers. . $^{30,33,34}$ In Greece, S. enterica serovar 1,4,[5],12:i:- was firstly recorded from humans in 2007 , accounting for only $0.3 \%$ of human cases of salmonellosis; its rate of isolation increased sharply thereafter, ranking third in frequency since 2009. In an EFSA report for the EU-wide baseline survey on the prevalence of Salmonella in slaughter pigs, $S$. serovar 1,4,[5],12:i:- was among the 'top five' serovars in Greece accounting for the $2.7 \%$ of the isolates. ${ }^{27}$ In addition, rarer serovars and subspecies, such as $S$. enterica 6,7:k:- and $S$. enterica subsp. diarizonae 61:k:1,5 were also identified in the present investigation. The source of subspecies diarizonae infecting man is thought to be reptiles kept as pets. ${ }^{35}$ This subspecies is considered host adapted to sheep, colonizing their nasal mucosa. ${ }^{36}$ Its isolation from pigs could indicate an emerging serovar for pork consumers and the same could be the cases with subspecies $S$. enterica subsp. houtenae, which, like the previous one, has traditionally been considered a subspecies infecting exotic reptiles, ${ }^{37}$ raptors $^{38}$ and humans only when coming in contact with these animal species. ${ }^{39}$ Such hosts pass in the environment their strains infecting food producing animals and eventually exposing consumers. Thus, physicians treating Salmonella cases caused by such exotic serovars, including those of subspecies diarizonae and salamae also found among Greek pigs, ${ }^{9,40}$ should include pork products in the possible sources of newly emerging exotic serovars or rarer subspecies.

The four (4) isolates $(6.67 \%)$ characterized during serotyping as 'rough', were considered isolates which had changed their surface antigens, thus were untypeable serologically. Such strains have developed surface mechanisms to evade host immune response and they are making their ' $\mathrm{O}$ ' antigens. ${ }^{41}$ Nevertheless, since all Salmonella serovars are considered potentially pathogenic for humans, the current observed wide distribution of serovars among pigs, implicate pork products to consumer infections not only by commonly recognized serovars, but also by a variety of rare ones. Salmonella spp. serovars, subclinically persisting in the tissue of pigs, ${ }^{4}$ make them a source of infection for other animals, the slaughter house environment and other carcasses, eventually reaching pork consumers. ${ }^{12,42}$ These epidemiological events are the same regardless of the prevalence of a serovar. However, the least prevalent will take longer before their isolation frequency increases among consumers to the point of concern. Thus, such rare serovars infecting pigs should be considered as "emerging" for Public Health and recorded as such instead of reported as "exotic", when isolated from human salmonellosis. Their rare isolation from food producing animals could rather reflect the sampling site. Feacal samples are traditionally the samples used for confirming a positive clinical or subclinical case. However, as it is evident (Table 1), from the 52 isolates only $11(21.1 \%)$ derived from feaces. As previously reported ${ }^{43,44}$ the best sites for confirming infection are the mucosal of the ileum and the mesenteric lymph nodes and this is possible only during carcass processing. In the present study 35 of the 52 isolates derived from the ileum (18 isolates) and mesenteric lymph nodes (17 isolates). In addition, due to that only nine animals were positive to more than one site; concurrent multiple-site carcass sampling should be considered as giving reliable results. Such a sampling protocol could also give information on serovars possibly co-existing in the same host. This is rarely reported ${ }^{45}$ either because it rarely happens or the serotyping procedure is limited, due to costs, to only phenotypically different isolates. However, in one instance of the present investigation two different serovars were identified from different carcass sampling sites of the same animal: serovar 6,7:k:- from the lymph nodes and serovar Infantis from feaces. Unfortunately, such multiple site isolations are impossible from human salmonellosis, thus it's unknown if one or more serovars cause an observed clinical case.

Furthermore, colonization of lymphatic tissue increases the risk to meat consumers, if cooking or handling does not eliminate the pathogen. This increased risk from lymphatic tissue colonization rather than carcass contamination during handling was evident in the present investigation through the examined meat and environmental samples. Six (6) of the muscle neck samples (site of carcass drainage) were positive to Salmonella and of them two came from a carcass that had Salmonella in their feaces, although all derived from animals with another positive site. As for the environmental samples considered evidence of possible slaughterhouse carcass contamination, the present investigation does not confirm other studies. ${ }^{46,47}$ Specifically, Salmonella was repeatedly isolated from the non - disposable gloves of only one of the skinners. Thus, hygiene measures were largely effective in the examined facilities, with the exemption of this worker's gloves. This type of gloves should be changed between handlings and meticulously cleaned. The serovars identified were Typhimurium and $S$. enterica subsp enterica ser 4,5,12:1-, both potential consumer pathogens, if passed to carcasses. Although the worker was not handling carcasses, he was part of the "house flora", which could contaminate carcasses, if HACCP procedures fail due to workers' negligence.

Table I Salmonella serovars found at five different bacteriological sampling sites of finishers

\begin{tabular}{llllll}
\hline Serovar & Feaces No & Ileum No & Lymph nodes No & Pig meat No & $\begin{array}{l}\text { Total isolates } \\
\text { per serovar }\end{array}$ \\
\hline S. Typhimurium & 6 & 7 & 2 & 5 & 20 \\
S.enterica subsp. enterica ser. 4,5,12:i:- & - & 5 & 0 & 1 & 6 \\
S.enterica subsp. enterica ser. 4,12:i:- & - & 2 & 5 & - & 7 \\
S. Bredeney & - & - & 2 & - & 2 \\
S. Agona & - & - & 1 & - & 1 \\
S. Derby & - & 1 & - & - & 1 \\
S.enterica subsp. enterica. 6,7:k:- & 2 & - & 4 & - & 6 \\
S. Infantis & 1 & - & - & - & 1 \\
\hline
\end{tabular}


Table Continued

\begin{tabular}{|c|c|c|c|c|c|}
\hline Serovar & Feaces No & Ileum No & Lymph nodes No & Pig meat No & $\begin{array}{l}\text { Total isolates } \\
\text { per serovar }\end{array}$ \\
\hline S. Meleagridis & 1 & - & - & - & 1 \\
\hline S. Cerro & - & 1 & - & - & 1 \\
\hline S. enterica subsp. diarizonae $61: \mathrm{k}: 1,5$ & 1 & - & - & - & 1 \\
\hline S. enterica subsp. houtenae 40:g,t:- & - & - & 1 & - & 1 \\
\hline Rough & - & 2 & 2 & - & 4 \\
\hline Total & 11 & 18 & 17 & 6 & 52 \\
\hline
\end{tabular}

\section{Conclusion}

From the above one concludes that the pig, thus pork meat, is a potential source of, not only serovars accepted as commonly pathogenic to consumers, but also of other rarer serovars and subspecies. These, colonizing the lymphatics of subclinically infected pigs, could eventually infect multiple consumers, if meat cooking and handling methods fail to protect them. In addition, the present evidence showed that strict HACCP control effectively controls the building up of "house flora" having microbes pathogenic to consumers.

\section{Acknowledgements}

None.

\section{Conflict of interest}

The author declares that they do not have a commercial or other association that might pose a conflict of interest.

\section{References}

1. EFSA, European Food Safety Authority. The Community Summary Report on Trends and Sources of Zoonoses, Zoonotic Agents and Food-Borne Outbreaks in the European Union in 2008. EFSA Journal. 2010;8(1):23-25.

2. EFSA, European Food Safety Authority. EFSA assesses risk of Salmonella from pig meat. 2010.

3. EFSA, European Food Safety Authority. Scientific Opinion on an estimation of the public health impact of setting a new target for the reduction of Salmonella in turkeys. EFSA Journal. 2012;10(4):1-89.

4. Wood RL, Pospischil A, Rose R. Distribution of persistent Salmonella Typhimurium infection in internal organs of swine. Am J Vet Res. 1989;50(7):1015-1021.

5. Fedorka Cray PJ, Gray JT, Wray C. Salmonella infections in pigs. In: WrayC, Wray A, editors. Salmonella in Domestic animals, CAB International: Wallingford; 2000. pp. 191-207.

6. Carter PB, Collins FM. The route of enteric infection in normal mice. $J$ Exp Med. 1974;139(5):1189-1203.

7. Hohmann AW, Schmidt G, Rowley D. Intestinal colonization and virulence of Salmonella in mice. Infect Immun. 1978;22(3):763-770.

8. Liebler Tenorio EM, Pabst R. MALT structure and function in farm animals. Veterinary Research, 2006;37(3):257-280.

9. Evangelopoulou G, Filioussis G, Kritas S, et al. Colonisation of pig gallbladders with Salmonella species important to public health. Veterinary Record. 2014;176(7):102822.

10. Dlabac V, Trebichavsky I, Rehakova Z, et al. Pathogenicity and protective effect of rough mutants of Salmonella species in germ-free piglets. Infect Immun. 1997;65(12):5238-5243.

11. Gonzalez-Escobedo G, Gunn S. Gallbladder Epithelium as a Niche for
Chronic Salmonella Carriage. Infect Immun. 2013;81(8):2920-2930.

12. Berends BR, Van Knapen F, Snijders JM, et al. Identification and quantification of risk factors regarding Salmonella spp. on pork carcasses. Int J Food Microbiol. 1997;36(2-3):199-206.

13. Warriner K, Aldsworth TG, Kaur S, et al. Cross-contamination of carcasses and equipment during pork processing. Journal of Applied Microbiology. 2002;93(1):169-177.

14. Baptista FM, Dahl J, Nielsen LR. Factors influencing Salmonella carcass prevalence in Danish pig abattoirs. Prev Vet Med. 2010;95(34):231-238

15. Visscher CF, Klein G, Verspohl J, et al. Serodiversity and serological as well as cultural distribution of Salmonella on farms and in abattoirs in Lower Saxony, Germany. Int J Food Microbiol. 2011;146(1):44-51.

16. Swanenburg M, Berends BR, Urlings HA, et al. Epidemiological investigations into the sources of Salmonella contamination of pork. Berl Munch Tierarztl Wochenschr. 2001;114(9-10):356-359.

17. Hald T, Wingstrand A, Swanenburg M, et al. The occurrence and epidemiology of Salmonella in European pig slaughterhouses. Epidemiol Infect. 2003;131(3):1187-1203.

18. Smith RP, Clough HE, Cook AJ. Analysis of Meat Juice ELISA Results and Questionnaire Data to Investigate Farm-Level Risk Factors for Salmonella Infection in UK Pigs. Zoonoses Public Health. 2010;57(S1):39-48.

19. ISO 6579:2002/Amd 1:2007. Microbiology of food and animal feeding stuffs: Horizontal method for the detection of Salmonella spp. Amendment 1: Annex D: Detection of Salmonella spp. in animal faeces and in environmental samples from the primary production stage. 2007.

20. Lo Fo Wong DM, Dahl J, Stege H, van der Wolf PJ, et al. Herd-level risk factors for subclinical Salmonella infection in European finishing-pig herds. Prev Vet Med. 2004;62(4):253-266.

21. Grafanakis E, Leontides L, Genigeorgis C. Seroprevalence and antibiotic sensitivity of serotypes of Salmonella enterica in Greek pig herds. Vet Rec. 2001;148(13):407-411.

22. Filioussis G, Petridou E, Johansson A, et al. Antimicrobial susceptibility and genetic relatedness of Salmonella enterica subsp. enterica serovar Mbandaka strains, isolated from a swine finishing farm in Greece. African Journal of Microbiology Research. 2008;2(11):313-315.

23. Pires SM, de Knegt L, Hald T. SCIENTIFIC / TECHNICAL REPORT submitted to EFSA: Estimation of the relative contribution of different food and animal sources to human Salmonella infections in the European Union. National Food Institute: Technical University of Denmark; 2011.

24. CDC, Centers for Disease Control and Prevention. Investigation of outbreak of human infections caused by Salmonella serotype I 4,[5],12:i:-. 2007.

25. EFSA, European Food Safety Authority. Scientific opinion on monitoring and assessment of the public health risk of "Salmonella Typhimurium-like" strains. EFSA Journal. 2010;8(10):1-48. 
26. Hopkins KL, Kirchner M, Guerra B, et al. Multiresistant Salmonella enterica serovar 4,[5],12:i:- in Europe: a new pandemic strain? Euro Surveillance. 2010;15(22):19580.

27. Mandilara G, Lambiri M, Polemis M, et al. Phenotypic and molecula characterisation of multiresistant monophasic Salmonella Typhimurium (1,4,[5],12:i:-) in Greece, 2006 to 2011. Euro Surveill. 18(22):pii=20496.

28. Moreno Switt AI, Soyer Y, Warnick LD, et al. Emergence, Distribution, and Molecular and Phenotypic Characteristics of Salmonella enterica Serotype 4,5,12:i:-. Foodborne Pathogens and Disease. 2009;6(4):407415.

29. Bone A, Noel H, Le Hello S, et al. Nationwide outbreak of Salmonella enterica serotype 4,12:i:- infections in France, linked to dried pork sausage. Euro Surveill. 2010;15(24):pii=19592.

30. Hauser E, Tietze E, Helmuth R, et al. Pork Contaminated with Salmonella enterica Serovar 4,[5],12:i:, an Emerging Health Risk for Humans. Appl Environ Microbiol. 2010;76(14):4601-4610.

31. EFSA, European Food Safety Authority. The European Union Summary Report on Trends and Sources of Zoonoses, Zoonotic Agents and Foodborne Outbreaks in 2011. EFSA Journal. 2013;11(4):3129.

32. EFSA (European Food Safety Authority), ECDC (European Centre for Disease Prevention and Control). The European Union Summary Report on antimicrobial resistance in zoonotic and indicator bacteria from humans, animals and food in 2011. EFSA Journal. 2013;11(5):3196.

33. Soyer Y, Moreno Switt A, Davis MA, et al. Salmonella enterica Serotype 4,5,12:i:-, an Emerging Salmonella Serotype That Represents Multiple Distinct Clones. J Clin Microbiol. 2009;47(11):3546-3556.

34. Bugarel M, Vignaud ML, Moury F, et al. Molecular identification in monophasic and nonmotile variants of Salmonella enterica serovar Typhimurium. Microbiologyopen. 2012;1(4):481-489.

35. Schröter M, Roggentin P, Hofmann J, et al. Pet snakes as a reservoir for Salmonella enterica subsp. diarizonae (Serogroup IIIb): a prospective study. Appl Environ Microbiol. 2004;70(1):613-615.

36. Lacasta D, Ferrer LM, Ramos JJ, Chronic Proliferative Rhinitis associated with Salmonella enterica subspecies diarizonae serovar 61:k:1, 5, (7) in Sheep in Spain. J Comp Pathol. 2012;147(4):406-409.

37. Bauwens L, Vercammen F, Bertrand S, et al. Isolation of Salmonella from environmental samples collected in the reptile department of
Antwerp Zoo using different selective methods. J Appl Microbiol. 2006;101(2):284-289.

38. Millán J, Aduriz G, Moreno B, et al. Salmonella isolates from wild birds and mammals in the Basque Country (Spain). Revue scientifique et technique. 2004;23(3):905-911.

39. Tabarani CM, Bennett NJ, Kiska DL, et al. Empyema of preexisting subdural hemorrhage caused by a rare Salmonella species after exposure to bearded dragons in a foster home. Journal of Pediatrics. 2010;156(6):322-323.

40. Evangelopoulou G, Kritas S, Govaris A, et al. Pork Meat as a Potential Source of Salmonella enterica subsp. arizonae Infection of Man. J Clin Microbiol. 2014;52(3);741-744.

41. Chandra M, Singh BR, Shankar H, et al. Study on prevalence of Salmonella infection in goats. Small Ruminant Research. 2006;65(12):24-30.

42. Vieira-Pinto M, Temudo P, Martins C. Occurrence of Salmonella in the Ileum, Ileocolic Lymph Nodes, Tonsils, Mandibular Lymph Nodes and Carcasses of Pigs Slaughtered for Consumption. J Vet Med B Infect Dis Vet Public Health. 2005;52(10):476-481.

43. Gray JT, Fedorka Cray PJ, Stabel TJ. Ackermann MR. Influence of inoculation route on the carrier state of Salmonella Choleraesuis in swine. Vet Microbiol. 1995;47(1-2):43-59.

44. Bahnson PB, Kim JY, Weigel RM, et al. Associations between on-farm and slaughter plant detection of Salmonella in market-weight pigs. $J$ Food Prot. 2005;68(2):246-250.

45. Garrido V, Sánchez S, San Román B, et al. Simultaneous infections by different Salmonella strains in mesenteric lymph nodes of finishing pigs. BMC Vet Res. 2014;10:59.

46. Limpitakis N, Genigeorgis C, Abrahim A, et al. Post-harvest epidemiology of Salmonella enterica in pork: Prevalence in the environment, carcasses and by-products in two slaughterhouses in Greece (1996-1998). Proceedings of the 3rd International Symposium on Epidemiology and Control of Salmonella in Pork: Washington; 1999. pp. 141-150.

47. Swanenburg M, Urlings HA, Snijders JM, et al. Salmonella in slaughter pigs: prevalence serotypes and critical control points during slaughter in two slaughterhouses. Int J Food Microbiol. 2001;70(3):243-254. 